\title{
Optical Imaging of Ionizing Radiation from Clinical Sources
}

\author{
Travis M. Shaffer ${ }^{1-4}$, Charles Michael Drain ${ }^{3,4}$, and Jan Grimm \\ ${ }^{1}$ Molecular Pharmacology Program, Memorial Sloan Kettering Cancer Center, New York, New York; ${ }^{2}$ Department of Radiology, \\ Memorial Sloan Kettering Cancer Center, New York, New York; ${ }^{3}$ Department of Chemistry, Hunter College of City University of New \\ York, New York, New York; ${ }^{4}$ Department of Chemistry, Graduate Center of City University of New York, New York, New York; \\ ${ }^{5}$ Department of Pharmacology, Weill Cornell Medical College, New York, New York; and ${ }^{6}$ Department of Radiology, Weill Cornell \\ Medical College, New York, New York
}

\begin{abstract}
Nuclear medicine uses ionizing radiation for both in vivo diagnosis and therapy. lonizing radiation comes from a variety of sources, including $x$-rays, beam therapy, brachytherapy, and various injected radionuclides. Although PET and SPECT remain clinical mainstays, optical readouts of ionizing radiation offer numerous benefits and complement these standard techniques. Furthermore, for ionizing radiation sources that cannot be imaged using these standard techniques, optical imaging offers a unique imaging alternative. This article reviews optical imaging of both radionuclide- and beam-based ionizing radiation from highenergy photons and charged particles through mechanisms including radioluminescence, Cerenkov luminescence, and scintillation. Therapeutically, these visible photons have been combined with photodynamic therapeutic agents preclinically for increasing therapeutic response at depths difficult to reach with external light sources. Last, new microscopy methods that allow single-cell optical imaging of radionuclides are reviewed.
\end{abstract}

Key Words: ionizing radiation; imaging; optical; dosimetry; radiation therapy; Cerenkov

J Nucl Med 2016; 57:1661-1666

DOI: 10.2967/jnumed.116.178624

The interaction of ionizing radiation with matter is at the heart of nuclear medicine. The late 19th century saw the beginning of both nuclear medicine and radiology (and in a way radiotherapy) in the discoveries of radioactivity by Becquerel and x-rays by Röntgen. As the nuclear physics field matured throughout the first half of the 20th century, opportunities developed for noninvasive in vivo imaging using ionizing radiation in the form of PET, SPECT, and planar scanners. These modalities are used for most radiotracer imaging, but for certain applications, using the visible light photons resulting from ionizing radiation interactions with matter offers unique benefits. Conventional methods that combine

Received May 23, 2016; revision accepted Sep. 3, 2016.

For correspondence or reprints contact: Jan Grimm, Molecular Pharmacology Program, Memorial Sloan Kettering Cancer Center, 1275 York Ave., Room 2025,

New York, NY 10065

E-mail: grimmj@mskcc.org

Published online Sep. 29, 2016.

COPYRIGHT (c) 2016 by the Society of Nuclear Medicine and Molecular Imaging, Inc. optical and nuclear imaging including the radiolabeling of fluorescent entities followed by fluorescent excitation with a laser ( $($ ) will not be discussed as the mechanism of visible light output is through laser excitation.

To consider optical imaging of ionizing radiation, an understanding of the mechanisms of ionizing radiation interactions with matter is essential. These mechanisms are well understood in medical physics and molecular imaging and are reviewed in depth (2). As high-energy photons and charged particles traverse matter, they dissipate energy in a manner dependent on the energy of the photon or particle, and the atomic number and density of the surrounding matter. Photons at energies used in medical imaging interact with surrounding matter mainly through 4 mechanisms: photoelectric effect, Compton scattering, coherent scattering, and pair production. The photoelectric effect occurs when an electron is ejected from an atom via absorption of the energy of a high-energy photon, which disappears in the process. Compton scattering involves the transfer of a portion of the photon energy to an electron, with the electron ejected from the atom and the high-energy photon scattered at a lower energy in the process. The photoelectric effect predominates at relatively low photon energies and high atomic numbers of interacting matter. With both the photoelectric effect and the Compton scattering, an outer shell electron fills the vacancy created by the ejected electron resulting in visible or x-ray photon emission, which can be imaged. Coherent scattering is an elastic process and generally occurs when the energy of the radiation is small compared with the ionization energy of the material. Pair production is the creation of an elementary particle and its antiparticle, for example, creating an electron and positron, but requires high-energy photons of at least $1.022 \mathrm{MeV}$ and rarely occurs with medical isotopes.

Charged particles, such as those from $\beta$ - and $\alpha$-particleemitting radionuclides, can interact with surrounding matter through 5 mechanisms: excitation, ionization, bremsstrahlung, Cerenkov, and annihilation (positron only). Excitation results in an electron occupying an excited state, which will subsequently relax to the ground state. Ionization results in the ejection of an electron from an atom, similar to the photoelectric and Compton effect seen with high-energy photons. Bremsstrahlung occurs when a charged particle is attracted (or repulsed) by the 
positive atomic nucleus, altering the momentum of the charged particle and thus resulting in the release of the lost kinetic energy as emission of photons. The Cerenkov mechanism occurs when a charged particle travels faster than the velocity of light in a medium, resulting in visible photon emission, deemed Cerenkov luminescence (CL). Annihilation occurs when the antimatter of an electron, the positron, interacts with an electron, causing the electron-positron pair to annihilate and two $511-\mathrm{keV}$ photons to be emitted at approximately $180^{\circ}$ apart.

Both high-energy photons and charged particles can result in more complex interactions than presented here, such as the formation of Auger electrons and secondary ionization. Though these mechanisms can complicate interpretation of in vivo imaging, translatable ex vivo calibration methods and simulations in preclinical models can result in acceptable sensitivity and resolution for in vivo optical imaging.

\section{OPTICAL IMAGING OF $\gamma$-EMITTING RADIOTRACERS}

Because $\gamma$-emitting radiotracers allow for planar scintigraphic and tomographic SPECT imaging, alternative imaging techniques are not typically considered. However, other methods exist; for example, visible photon emission from $\gamma$-emitting radiotracers has been known in the nuclear physics field for decades (3). All ionizing radiation sources, such as the $140-\mathrm{keV}$ photons emitted by ${ }^{99 \mathrm{~m}} \mathrm{Tc}$, result in ionization and

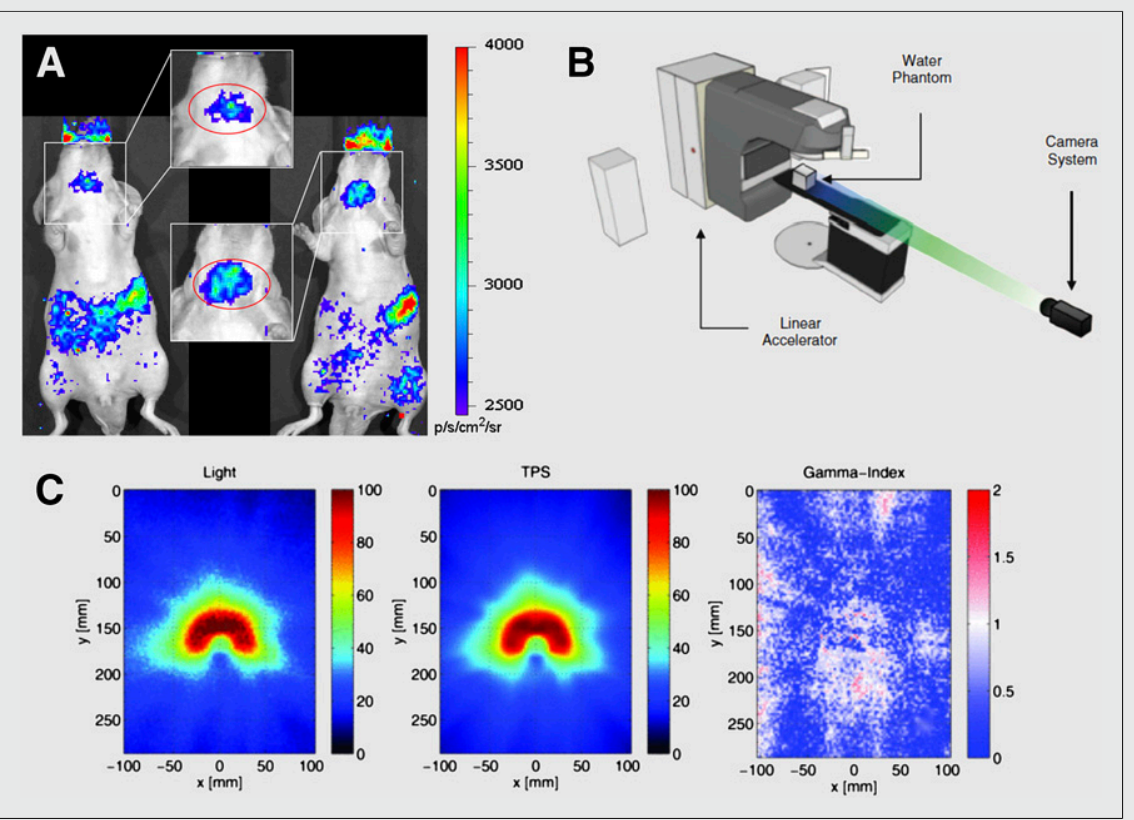

FIGURE 1. Optical imaging of $\gamma$-emitters and photon beams. (A) Radioluminescence image of $99 \mathrm{mTc}$-pertechnetate distribution in $\mathrm{Nu} / v$ mice showing salivary, thyroid, and stomach uptake. (B) Linear accelerator phantom study to image Cerenkov light from photon beam interactions with water. Distance of camera to water phantom is $3 \mathrm{~m}$. (C) Two-dimensional projections of experimentally imaged 3-dimensional (3D) Cerenkov light volume (left) and nonlinearly summed 3D dose matrix from TPS (center) are shown for the VMAT treatment plan. Resulting $\gamma$-index map is shown with a $3 \% / 3-\mathrm{mm}$ dose difference and DTA criterion (right). DTA = distance-to-agreement; TPS = treatment planning system; VMAT = volumetric-modulated arc therapy. (A and B-C adapted with permission of (6) and (9), respectively.) excitation of the medium, such as water. The relaxation of water molecules and transition of excited hydroxyl radicals result in the emission of visible light, known as radioluminescence. These visible photons were recently used to allow optical imaging of clinical $\gamma$-emitters. Optical imaging with ${ }^{99 \mathrm{~m} T c}$, the $\gamma$-emitter used widely for SPECT and planar imaging, was first shown using a phosphor screen that converted the high-energy photons into visible light, which was imaged in a preclinical optical IVIS system (PerkinElmer). Optical resolution is better than $1.3 \mathrm{~mm}$ when the source depth is less than $10 \mathrm{~mm}$, highlighting the depth dependence of optical methods (4). Subsequently, visible photons arising from the interactions of various ${ }^{99 \mathrm{~m} T c} \gamma$-systems with tissue were directly imaged, obviating a phosphor screen. Radioluminescence with variations in the emission spectrum was observed for each of the ${ }^{99 \mathrm{~m}} \mathrm{Tc}$ systems. The visible signal exhibited a half-life of 6-7 h, which corresponds to the half-life of ${ }^{99 \mathrm{~m} T c}(6.02 \mathrm{~h})$ and demonstrates that the visible light was from ${ }^{99 \mathrm{~m}} \mathrm{Tc}$ and not a contaminant in the ${ }^{99} \mathrm{~m}$ Tc solution. In vivo optical imaging was shown in a study with ${ }^{99 m}$ Tc-phytate colloids, but the benefits over SPECT imaging were not readily apparent (5). In another optical imaging demonstration, ${ }^{99 \mathrm{~m} T c-p e r t e c h n e t a t e}$ was used for optical imaging of the thyroid and salivary glands after uptake by the sodium-iodide symporter (Fig. 1A) (6).

Although visible light emission from SPECT radionuclides is an interesting phenomenon, the low flux of visible photons $\left(10^{4}\right.$ vs. $8 \times 10^{6}$ average radiance $\left[\mathrm{p} / \mathrm{s} / \mathrm{cm}^{2} / \mathrm{sr}\right]$ for scaled activity amounts of ${ }^{99 \mathrm{~m}} \mathrm{Tc}$ via radioluminescence and ${ }^{18} \mathrm{~F}$ via $\mathrm{CL}$, respectively) (7) makes in vivo optical imaging even more challenging than CL imaging (CLI). A variety of nanoparticles, deemed nanoscintillators, can enhance the visible photon output from $\gamma$-emitters and $\mathrm{x}$-rays; however, these systems are only in the preclinical stage and have significant barriers to translation, so will only be briefly discussed herein. High-energy photons interact with the nanoscintillators to excite electrons, which can relax both radiatively by emitting a visible photon and nonradiatively. Clinical feasibility is uncertain because toxicity issues arising from the composition, size, and biodistribution of the nanoparticles need to be investigated before applications. A detailed review on nanoscintillators in preclinical use was recently published (8).

\section{External High-Energy Photons}

High-energy photons also have external sources, such as photon beams or X-rays commonly used in clinical 
and preclinical practice. Clinical photon beams operate in the megavoltage region, resulting in ejection-from atomsof electrons with energies exceeding that of the Cerenkov threshold in tissue $(220 \mathrm{keV})$. The ejected electrons result in CL, allowing CLI (Figs. 1B and 1C). Both intensitymodulated radiation therapy and volumetrics-modulated arc therapy were evaluated with real-time video-rate optical dosimetry using CL (9). A more elaborate system involving linear accelerators is Cerenkov-excited fluorescence tomography, for which the generated CL excites fluorophores in a sample. Because the light from CL is blue-weighted, the use of fluorophores that emit in the red or near-infrared range allows deeper imaging depth at the cost of the increased complexity of injecting a dye before beam therapy $(10,11)$.

\section{OPTICAL IMAGING OF CHARGED PARTICLES}

\section{$\boldsymbol{\beta}$ - and $\boldsymbol{\alpha}$-Emitting Radiotracers: Cerenkov}

Optical imaging of $\beta$ - and $\alpha$-emitting radiotracers has been demonstrated through various mechanisms of energy dissipation by charged particles. CLI is the most common way that ionizing radiation is optically imaged for 3 reasons: CL is typically of significantly higher intensity than radioluminescence from $\gamma$-emitters; CL allows imaging of $\beta$ - and $\alpha$-emitters, which can be challenging to image; and CLI is possible with most clinical $\beta$-radiotracers, allowing rapid clinical translation. Because CL is blue lightweighted, and current clinical use of CLI relies only on a given radiotracer without endogenous enhancement entities, only imaging radionuclides close to the surface is

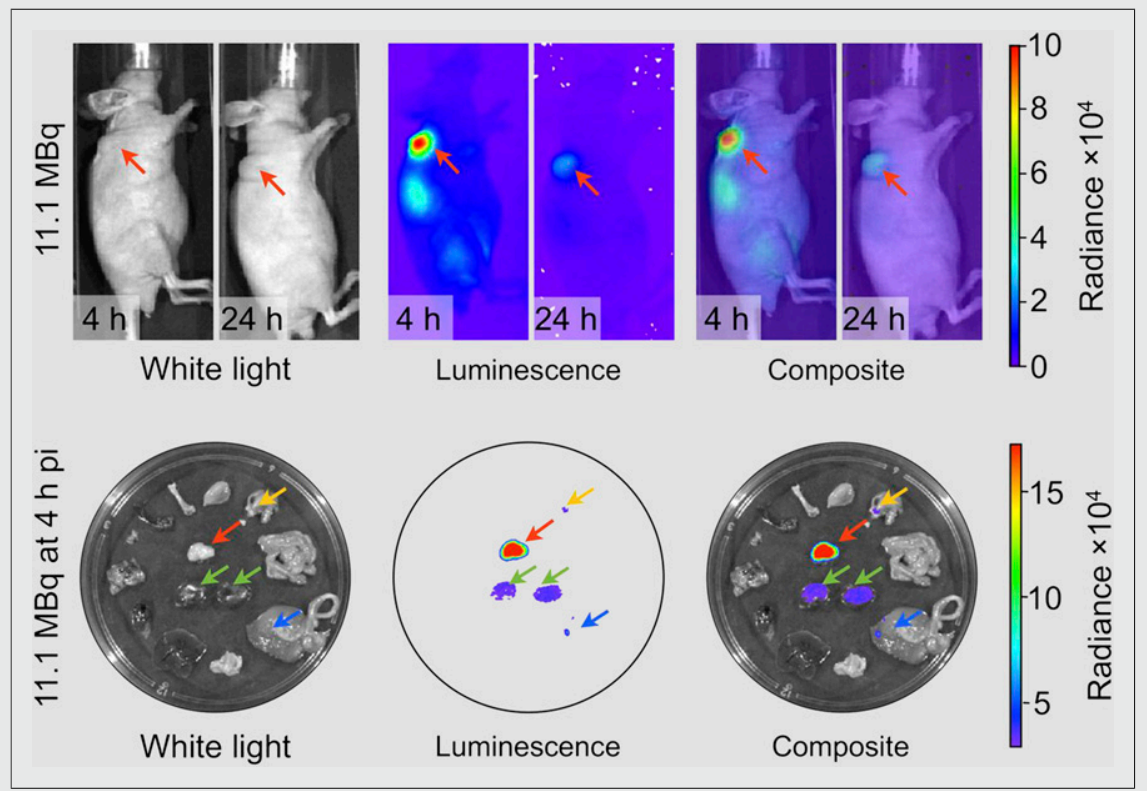

FIGURE 2. Cerenkov imaging of therapeutic radiotracers. (Top) CLI of ${ }^{90}$ Y-DOTA-AR in mice with PC-3 prostate cancer tumors. Tumors (red arrow) show high radiance at $4 \mathrm{~h}$ after injection (pi) with partial clearance at $24 \mathrm{~h}$ after injection. (Bottom) Ex vivo images at $4 \mathrm{~h}$ after injection, with tumor clearly delineated (red arrow). Green arrows = kidneys; yellow arrow = stomach; blue arrow = large intestine. (Adapted with permission of (17).) possible. To address this depth limitation, increased imaging depth has been achieved preclinically through pairing radiotracers with photoluminescence molecules or nanoparticles that absorb blue light and emit a more penetrating red (12). As these systems are further evaluated, clinical translation could allow improvements of radionuclide imaging, which is currently challenging.

The first CLI clinical studies used the PET tracer ${ }^{18} \mathrm{~F}$ FDG in endoscopic studies and detection of nodal disease $(13,14)$, whereas another study imaged ${ }^{131}$ I thyroid uptake in 1 patient (15). These proof-of-concept studies used widely applied radiotracers to correlate images obtained through traditional nuclear medicine instrumentation with images obtained through CLI as a means to quantify the efficacy. Conversely, a major benefit of CLI is the ability to image therapeutic radiotracers. Traditional detection methods for ${ }^{90} \mathrm{Y}$ (maximum energy of the electron [referred to as $\alpha$-particle], 2.28 MeV) are difficult because of the absence of suitable $\gamma$-emissions and low amount of positron emissions, but the high CL intensity, which is over 35 times that of ${ }^{18} \mathrm{~F}$ (16), makes ${ }^{90} \mathrm{Y}$ an ideal candidate for CLI. Recently in a preclinical model, it was shown that ${ }^{90} \mathrm{Y}$ could be quantitatively imaged using CLI (17). The ${ }^{90}$ Y-labeled gastrinreleasing peptide receptor antagonist DOTA-AR was injected into prostate cancer xenograft models and imaged out to $72 \mathrm{~h}$ after injection using CLI (Fig. 2A). Quantification of tumor uptake using CLI correlated with ex vivo measurements (Fig. 2B), thus offering means for dosimetry as well as an alternative to PET imaging of the low amount of positrons emitted from ${ }^{90} \mathrm{Y}$. Cetuximab labeled with either ${ }^{90} \mathrm{Y}$ or ${ }^{177} \mathrm{Lu}$ was also imaged ex vivo using CLI, again showing the utility of CL for radionuclides that are often difficult to image using traditional methods (18). Therapeutically, ${ }^{90} \mathrm{Y}$ was combined with a photodynamic therapy agent for a synergistic system of both radiotherapy and photodynamic therapy excited by CL (19). In an interesting demonstration of the clinical utility of CL, brain tumor specimens were optically imaged during brain surgery, showing the uptake of injected ${ }^{90}$ Y-DOTATOC (20). Another potential application of CLI is imaging of ${ }^{90} \mathrm{Y}$ microspheres used for radioembolization. In future studies, the microspheres could incorporate fluorescent molecules that absorb CL and emit in the nearinfrared range, allowing noninvasive near-infrared imaging of the spheres as an alternative to current methods of PET/CT imaging and SPECT bremsstrahlung imaging (21).

$\alpha$-emitters such as ${ }^{213} \mathrm{Bi}$ and ${ }^{225} \mathrm{Ac}$ are used therapeutically through conjugation 
to antibodies, nanomaterials, and peptides $(22,23)$. Because these radionuclides emit an $\alpha$-particle, conventional imaging can be achieved only using indirect methods, such as the injection of an imaging radiotracer attached to the same targeting ligand, followed by injection of the $\alpha$-emitter, with the assumption that the biodistribution is similar. Unfortunately, the energy of the $\alpha$-particle is too low to allow direct CL. However, the $\alpha$-decay can be indirectly visualized with CLI of the $\beta$-emitting daughter decay products of ${ }^{225} \mathrm{Ac}$, which generate CLI (22). Another application of this approach was recently demonstrated. ${ }^{225}$ Ac-DOTA-c(RGDyK) was injected into U87mg human glioblastoma xenograft mice and CLI conducted at $24 \mathrm{~h}$ after injection (24). As $\alpha$-emitters continue to be investigated as radiotherapeutics, imaging strategies such as CLI should prove useful for dosimetry and radionuclide localization.

One ideal application for optical imaging of ionizing radiation is endoscopy of radiotracers that emit CL, coined $\mathrm{CL}$ endoscopy. Interference from ambient light is not a significant issue with endoscopic imaging. The applicability of this technique was shown preclinically with ${ }^{18}$ F-FDG using an optical fiber bundle connected to a charge-coupled device (25) and later with both ${ }^{90} \mathrm{Y}$ and ${ }^{18} \mathrm{~F}(26)$, with both studies using subcutaneous tumor models. CL endoscopy was rapidly translated to the clinic for distinguishing and quantifying cancerous lesions of the gastrointestinal tract in patients, just $3 \mathrm{y}$ after the preclinical studies (13). Here the clinical radiotracer ${ }^{18} \mathrm{~F}-\mathrm{FDG}$ was used and PET/CT-positive lesions corresponded with positive legions using CL endoscopy; however, the imaging was obtained only in a contact mode, with the endoscope in contact with the lesion.

\section{$\beta-$ and $\alpha$-Emitting Radiotracers: Scintillation}

Although CL is widely used for in vivo optical imaging, scintillation offers another route for charged particle optical imaging. Here, a material is used that produces optical photons upon excitation by ionizing radiation. In an endoscopic application, a scintillation balloon was used to image atherosclerotic plaques in diabetic, hyperlipidemic mice injected with ${ }^{18} \mathrm{~F}-\mathrm{FDG}$ (27). The left common carotid artery was ligated whereas the nonligated right carotid was the control. When a scintillating balloon-enabled fiber-optic radionuclide imaging system was used, the ligated artery was shown to have an increased ${ }^{18} \mathrm{~F}-\mathrm{FDG}$ uptake compared with the control, corresponding with ex vivo autoradiography. The scintillating balloon system has a resolution of $1.2 \mu \mathrm{m}$, thereby overcoming some of the issues with PET imaging of atherosclerotic plaques such as the small size of plaques and ${ }^{18} \mathrm{~F}$-FDG uptake obscuration by adjacent myocardium.

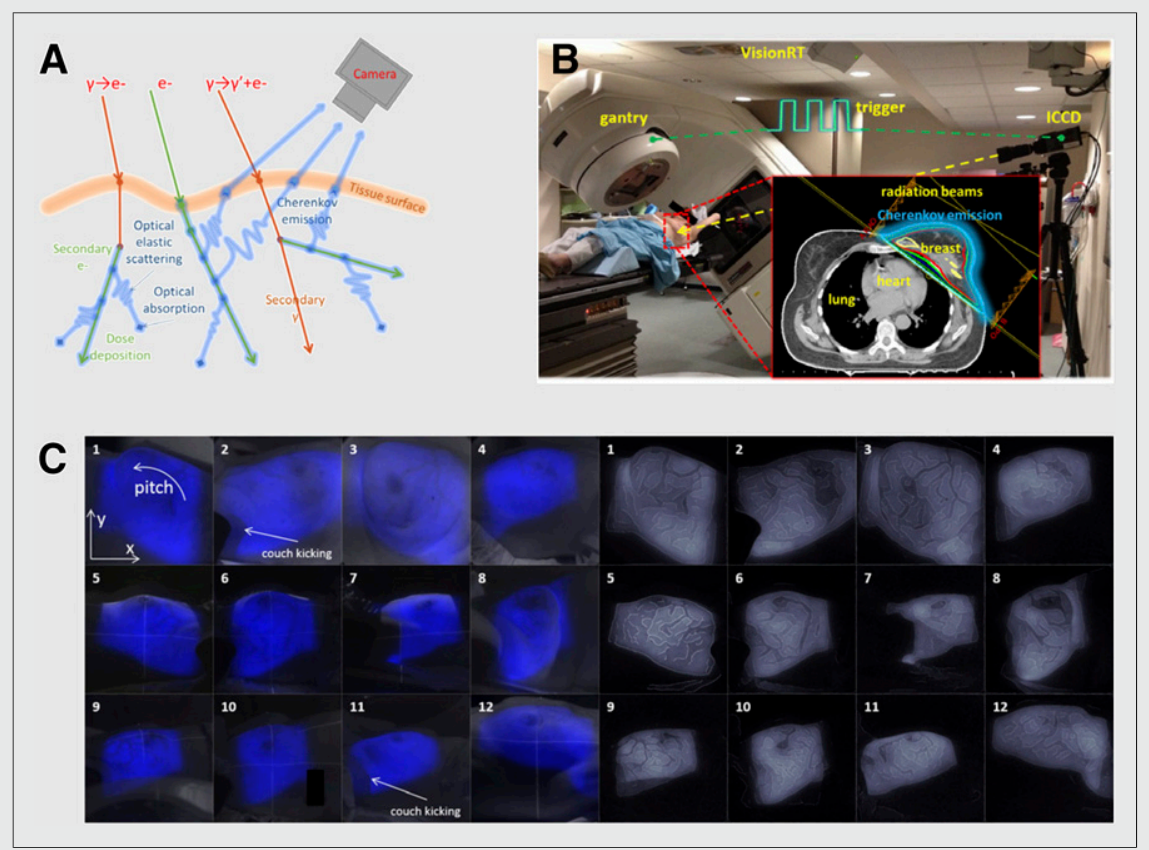

FIGURE 3. Optical imaging of charged particle beams. (A) Various interactions that may occur during charged particle beam therapy, including primary Cerenkov production, secondary Cerenkov, and various scattering and absorption processes. (B) Imaging of a patient undergoing postlumpectomy radiation therapy (inset, plan) using time domain-gated imaging system, which is synchronized to delivered radiation pulses. (C) Cerenkov images of each patient and corresponding whitelight images (left). Edge-enhanced Cerenkov images for each patient (right). (Adapted with permission of (28), (c) Institute of Physics and Engineering in Medicine.)

\section{Electron and Proton Beams}

Proton and electron beams are of increasing interest for radiation therapy. At the energies typically used ( $\mathrm{keV}$ through $\mathrm{MeV}$ ), they also produce Cerenkov light (Fig. 3A). In a phase I study, 12 patients undergoing postlumpectomy whole-breast irradiation from a linear accelerator were imaged in real time using CL (Fig. 3A) (28), thereby demonstrating that CLI can be used for monitoring treatment, positioning patients, tracking motion, and examining posttreatment results (Figs. 3B and 3C). Recently, total skin electron beam therapy for treatment of cutaneous lymphomas was monitored with CLI, and significantly lower doses were found applied to the legs than the torso, indicating a direct clinical relevance (29). In an exciting combination of clinical beam therapy and preclinical advances, the CL from external-beam therapy was combined with titanium dioxide nanoparticles to increase the therapeutic potential of beam therapy (30). The use of CL for excitation of nanophotodynamic therapy agents was previously shown with radiotracers 
$\left({ }^{18} \mathrm{~F}\right.$ and $\left.{ }^{64} \mathrm{Cu}\right)(31)$, highlighting the fact that different sources of ionizing radiation can be used for this application.

Proton beams increasingly used in radiation therapy offer unique imaging opportunities. For example, the positron emitters ${ }^{15} \mathrm{O}$ and ${ }^{11} \mathrm{C}$ are generated during proton beam therapy, which can enable PET/CT or Cerenkov imaging of patients to determine dose and location. PET/CT after proton irradiation was performed for 9 patients to image the generated isotopes, with imaging started within $20 \mathrm{~min}$ of proton beam therapy. PET/CT imaging results showed an average quantitative agreement of 5\%-30\% between calculated and measured PET distributions in the space and time domains (32). Because both ${ }^{15} \mathrm{O}$ and ${ }^{11} \mathrm{C}$ are positron emitters with relatively high energies, both result in appreciable CL emission, which was imaged with high resolution in proton beam tissue phantoms (33).

\section{NEW MICROSCOPY OPPORTUNITIES}

Microscopy offers superior resolution, sensitivity, and costaining of biomarkers compared with standard in vivo imaging. For ex vivo radionuclide imaging, autoradiography is typically used on tissue samples and slices, with the resolution dependent on the energy of the emitted radiotracer. A new method of ex vivo detection of ionizing radiation was demonstrated and deemed radioluminescence microscopy (RLM). RLM combines a sample containing ionizing radiation with a scintillator plate wherein the

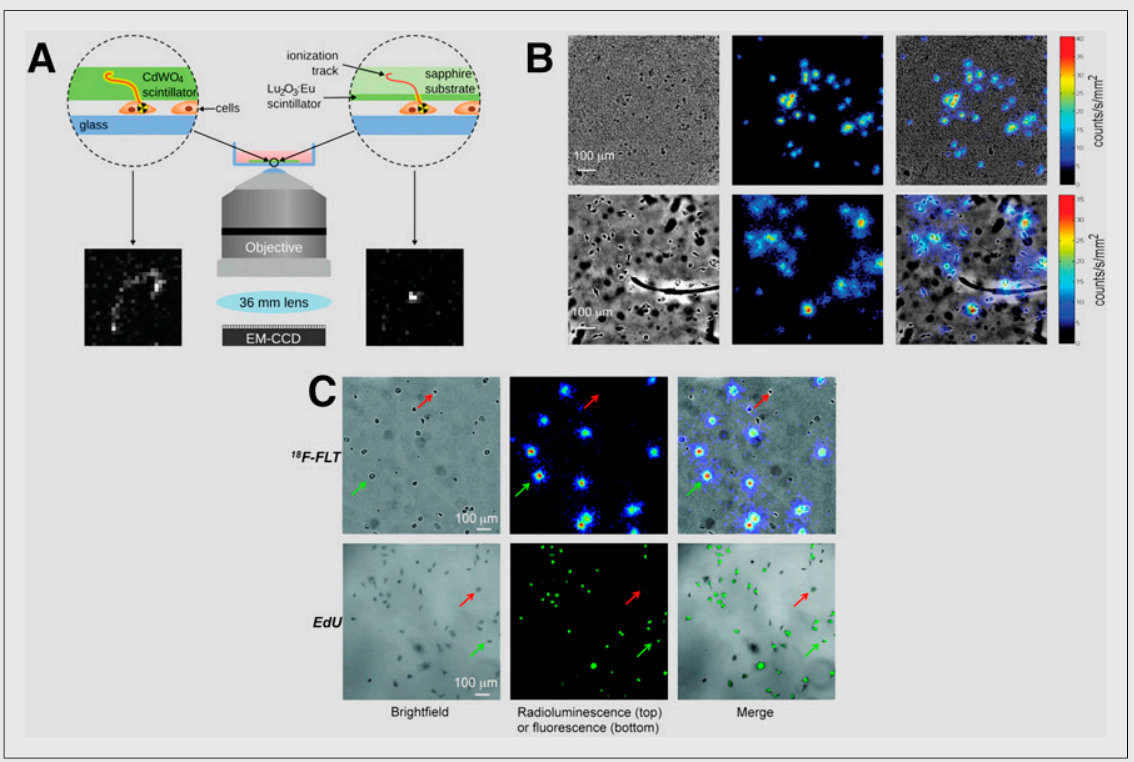

FIGURE 4. Radioluminescence microscopy. (A) Choice of scintillator matters. Scheme of radioluminescence microscopy setup comparing $500-\mu \mathrm{m} \mathrm{CdWO}_{4}$ scintillator (left) and $10-\mu \mathrm{m} \mathrm{Lu}_{2} \mathrm{O}_{3}:$ Eu scintillator (right). Because of thinness of $\mathrm{Lu}_{2} \mathrm{O}_{3}: E u$ scintillator, ionization tracks are shorter than with thicker $\mathrm{CdWO}_{4}$ scintillator. (B) Comparison of $\mathrm{Lu}_{2} \mathrm{O}_{3}: \mathrm{Eu}$ (top) and $\mathrm{CdWO}_{4}$ (bottom) scintillators with ${ }^{18} \mathrm{~F}-\mathrm{FDG}$, showing bright-field, radioluminescence, and overlaid images. (C) MDA-MB-231 cells imaged using ${ }^{18} \mathrm{~F}-$ FLT and radioluminescence microscopy (top) or 5-ethynyl-2'-deoxyuridine (EdU) and fluorescence microscopy (bottom). Red and green arrows indicate cells with negative and positive signals, respectively. (A-B and $C$ adapted with permission of (36) and (37), respectively.) interactions between the two result in visible photons that are imaged using a standard fluorescence microscope. In the first demonstration of RLM, single-cell resolution was demonstrated with live cells cultured on a 100-mm-thin $\mathrm{CdWO}_{4}$ scintillator plate with ${ }^{18}$ F-FDG (34). Subsequent work led to improvements in signal-to-background ratio through the use of optical reconstruction of the $\beta$-ionization track techniques led to increased sensitivity and resolution with no dependency on the $\beta$-particle energy (35). RLM hardware improvements were also investigated through the use of a $\mathrm{Lu}_{2} \mathrm{O}_{3}: \mathrm{Eu}$ scintillation plate $(6-10 \mu \mathrm{m}$ thick) that had increased sensitivity and resolution compared with a conventional scintillation plate composed of $\mathrm{CdWO}_{4}$ (500 $\mu \mathrm{m}$ thick) (Figs. 4A and 4B). The thinner $\mathrm{Lu}_{2} \mathrm{O}_{3}: \mathrm{Eu}$ plate has a higher photon yield and emissions in the greenred region of the visible spectrum, where typical chargecoupled devices have greater quantum efficiency compared with blue emissions by $\mathrm{CdWO}_{4}(36)$. In a practical demonstration of RLM, 3'-deoxy-3' - ${ }^{18}$ F-fluorothymidine ( ${ }^{18} \mathrm{~F}$-FLT) uptake was imaged in MDA-MB-231 cells grown under different serum conditions and compared with fluorescence microscopy of 5-ethynyl-2'-deoxyuridine incorporation in fixed cells, a standard measure of cell proliferation (Fig. 4C) (37). Under serum deprivation, few cells $(1 \%)$ incorporated 5ethynyl-2'-deoxyuridine whereas ${ }^{18}$ F-FLT uptake was low, but above background and heterogeneous, with a subset of cells being positive. Under $20 \%$ serum conditions, the frequency distribution of ${ }^{18} \mathrm{~F}$-FLT uptake in single cells was bimodal, with $61 \%$ of cells in the ${ }^{18}$ F-FLThigh subpopulation. RLM allows imaging of radiolabeled molecules on living cells at a single-cell level, enabling examination of individual cellular uptake rather than average uptake in a whole population.

Although the prior examples were completed using in vitro samples, RLM was also used with ex vivo tissue samples, directly analogous to autoradiography (38). Here, ${ }^{89} \mathrm{Zr}$-rituximab was used to image human CD20 expression in a huCD20 transgenic mouse model, which expresses CD20 on B cells. PET imaging of ${ }^{89} \mathrm{Zr}$-rituximab showed uptake in the spleen as expected, and ex vivo RLM and autoradiography both showed ${ }^{89} \mathrm{Zr}$-rituximab in the red pulp on the spleen. A comparison between RLM and conventional storage-phosphor autoradiography demonstrated that the former has superior sensitivity and resolution, with RLM requiring $80 \%$ fewer decays for imaging with a magnification of 4×. RLM's advantages compared with conventional autoradiography 
make it attractive for applications in which sensitivity and resolution are important, such as for imaging of radiolabeled tracers when there is cell heterogeneity, and for simultaneous imaging of both fluorescence and radioluminescence.

\section{CONCLUSION}

Ionizing radiation is an intrinsic component of nuclear medicine and radiology. Optical imaging of ionizing radiation interactions with matter has been demonstrated in both preclinical models and clinically and is moving increasingly into the focus of ongoing research. Visible light can be used for imaging and dosimetry with injected radiotracers or beams, allowing a broad range of applications including dosimetry, imaging, and therapy. The use of injected agents to enhance visible photon flux from ionizing radiation is common, although this approach leads to increasing complexity because of the use of an exogenous agent. Chargecoupled devices with higher quantum efficiency outside the typical 400-900 $\mathrm{nm}$ will allow superior sensitivity to both blue-weighted and near-infrared-emitting agents. Clinically, optical imaging of therapeutic tracers such as $\beta$ - and $\alpha$-emitters enables alternatives to current methods of dosimetry and anatomic placement. A thorough understanding of ionizing radiation interactions with matter is necessary, and collaborations between medical physics and imaging researchers are essential for the field to progress.

\section{DISCLOSURE}

This work was supported by the following grants: National Institutes of Health (NIH) R01EB014944 and R01CA183953 (to Jan Grimm) and National Science Foundation (NSF) Integrative Graduate Education and Research Traineeship grant DGS 0965983 (to Charles Michael Drain). No other potential conflict of interest relevant to this article was reported.

\section{REFERENCES}

1. Verbeek FP, Tummers QR, Rietbergen DD, et al. Sentinel lymph node biopsy in vulvar cancer using combined radioactive and fluorescence guidance. Int $J$ Gynecol Cancer. 2015;25:1086-1093.

2. Gupta TK. Radiation, Ionization, and Detection in Nuclear Medicine. Berlin, Germany: Springer Verlag; 2013.

3. Sitharamarao DN, Duncan JF. Molecular excitation of water by $\gamma$-irradiation. J Phys Chem. 1963;67:2126-2132.

4. Boschi F, Spinelli AE, D'Ambrosio D, Calderan L, Marengo M, Sbarbati A. Combined optical and single photon emission imaging: preliminary results. Phys Med Biol. 2009;54:L57-L62.

5. Kondakov AK, Gubskiy IL, Znamenskiy IA, Chekhonin VP. Possibilities of optical imaging of the ${ }^{99 \mathrm{~m}}$ Tc-based radiopharmaceuticals. J Biomed Opt. 2014;19:046014.

6. Boschi F, Pagliazzi M, Rossi B, et al. Small-animal radionuclide luminescence imaging of thyroid and salivary glands with Tc99m-pertechnetate. J Biomed Opt. 2013;18:76005.

7. Spinelli AE, Lo Meo S, Calandrino R, Sbarbati A, Boschi F. optical imaging of Tc-99m-based tracers: in vitro and in vivo results. J Biomed Opt. 2011;16:116023.

8. Kamkaew A, Chen F, Zhan Y, Majewski RL, Cai W. Scintillating nanoparticles as energy mediators for enhanced photodynamic therapy. ACS Nano. 2016;10:3918-3935.

9. Glaser AK, Andreozzi JM, Davis SC, et al. Video-rate optical dosimetry and dynamic visualization of IMRT and VMAT treatment plans in water using Cherenkov radiation. Med Phys. 2014;41:062102.

10. Demers JL, Davis SC, Zhang R, Gladstone DJ, Pogue BW. Cerenkov excited fluorescence tomography using external beam radiation. Opt Lett. 2013;38:1364-1366.
11. Glaser AK, Davis SC, Voigt WH, Zhang R, Pogue BW, Gladstone DJ. Projection imaging of photon beams using Cerenkov-excited fluorescence. Phys Med Biol. 2013;58:601-619.

12. Tanha K, Pashazadeh AM, Pogue BW. Review of biomedical Cerenkov luminescence imaging applications. Biomed Opt Express. 2015;6:3053-3065.

13. Hu H, Cao X, Kang F, et al. Feasibility study of novel endoscopic Cerenkov luminescence imaging system in detecting and quantifying gastrointestinal disease: first human results. Eur Radiol. 2015;25:1814-1822.

14. Thorek DL, Riedl CC, Grimm J. Clinical Cerenkov luminescence imaging of ${ }^{18}$ F-FDG. J Nucl Med. 2014;55:95-98.

15. Spinelli AE, Ferdeghini M, Cavedon C, et al. First human Cerenkography. J Biomed Opt. 2013;18:20502.

16. Gill RK, Mitchell GS, Cherry SR. Computed Cerenkov luminescence yields for radionuclides used in biology and medicine. Phys Med Biol. 2015;60:4263-4280.

17. Lohrmann $\mathrm{C}$, Zhang $\mathrm{H}$, Thorek DL, et al. Cerenkov luminescence imaging for radiation dose calculation of a ${ }^{90} \mathrm{Y}$-labeled gastrin-releasing peptide receptor antagonist. J Nucl Med. 2015;56:805-811.

18. Chakravarty R, Chakraborty S, Sarma HD, Nair KV, Rajeswari A, Dash A. ${ }^{90} \mathrm{Y} /{ }^{177} \mathrm{Lu}$-labelled cetuximab immunoconjugates: radiochemistry optimization to clinical dose formulation. J Labelled Comp Radiopharm. 2016;59:354-363.

19. Hartl BA, Hirschberg H, Marcu L, Cherry SR. Activating photodynamic therapy in vitro with Cerenkov radiation generated from yttrium-90. J Environ Pathol Toxicol Oncol. 2016;35:185-192.

20. Spinelli AE, Schiariti MP, Grana CM, Ferrari M, Cremonesi M, Boschi F. Cerenkov and radioluminescence imaging of brain tumor specimens during neurosurgery. J Biomed Opt. 2016;21:50502.

21. Zade AA, Rangarajan V, Purandare NC, et al. ${ }^{90} \mathrm{Y}$ microsphere therapy: does ${ }^{90} \mathrm{Y}$ PET/CT imaging obviate the need for ${ }^{90} \mathrm{Y}$ Bremsstrahlung SPECT/CT imaging? Nucl Med Commun. 2013;34:1090-1096.

22. Ruggiero A, Holland JP, Lewis JS, Grimm J. Cerenkov luminescence imaging of medical isotopes. J Nucl Med. 2010;51:1123-1130.

23. McDevitt MR, Ma D, Lai LT, et al. Tumor therapy with targeted atomic nanogenerators. Science. 2001;294:1537-1540.

24. Pandya DN, Hantgan R, Budzevich MM, et al. Preliminary therapy evaluation of ${ }^{225} \mathrm{Ac}$-Dota-C(Rgdyk) demonstrates that Cerenkov radiation derived from ${ }^{225} \mathrm{Ac}$ daughter decay can be detected by optical imaging for in vivo tumor visualization. Theranostics. 2016;6:698-709.

25. Liu H, Carpenter CM, Jiang H, et al. Intraoperative imaging of tumors using Cerenkov luminescence endoscopy: a feasibility experimental study. J Nucl Med. 2012;53:1579-1584.

26. Carpenter CM, Ma X, Liu H, et al. Cerenkov luminescence endoscopy: improved molecular sensitivity with $\beta$-emitting radiotracers. J Nucl Med. 2014;55:1905-1909.

27. Zaman RT, Kosuge H, Carpenter C, Sun C, McConnell MV, Xing L. Scintillating balloon-enabled fiber-optic system for radionuclide imaging of atherosclerotic plaques. J Nucl Med. 2015;56:771-777.

28. Zhang R, Andreozzi JM, Gladstone DJ, et al. Cherenkoscopy based patient positioning validation and movement tracking during post-lumpectomy whole breast radiation therapy. Phys Med Biol. 2015;60:L1-L14.

29. Andreozzi JM, Zhang R, Gladstone DJ, et al. Cherenkov imaging method for rapid optimization of clinical treatment geometry in total skin electron beam therapy. Med Phys. 2016;43:993-1002.

30. Ouyang Z, Liu B, Yasmin-Karim S, Sajo E, Ngwa W. Nanoparticle-aided external beam radiotherapy leveraging the Cerenkov effect. Phys Med. 2016;32:944-947.

31. Kotagiri N, Sudlow GP, Akers WJ, Achilefu S. Breaking the depth dependency of phototherapy with Cerenkov radiation and low-radianceresponsive nanophotosensitizers. Nat Nanotechnol. 2015;10:370-379.

32. Parodi K, Paganetti H, Shih HA, et al. Patient study of in vivo verification of beam delivery and range, using positron emission tomography and computed tomography imaging after proton therapy. Int J Radiat Oncol Biol Phys. 2007;68:920-934.

33. Yamamoto S, Toshito T, Fujii K, Morishita Y, Okumura S, Komori M. High resolution Cerenkov light imaging of induced positron distribution in proton therapy. Med Phys. 2014;41:111913.

34. Pratx G, Chen K, Sun C, et al. Radioluminescence microscopy: measuring the heterogeneous uptake of radiotracers in single living cells. PLoS One. 2012;7:e46285.

35. Pratx G, Chen K, Sun C, et al. High-resolution radioluminescence microscopy of ${ }^{18}$ F-FDG uptake by reconstructing the $\beta$-ionization track. J Nucl Med. 2013;54:1841-1846.

36. Sengupta D, Miller S, Marton Z, Chin F, Nagarkar V, Pratx G. Bright $\mathrm{Lu}_{2} \mathrm{O}_{3}: \mathrm{Eu}$ thin-film scintillators for high-resolution radioluminescence microscopy. $A d v$ Healthc Mater. July 16, 2015 [Epub ahead of print].

37. Sengupta D, Pratx G. Single-cell characterization of ${ }^{18}$ F-FLT uptake with radioluminescence microscopy. $J$ Nucl Med. 2016;57:1136-1140.

38. Natarajan A, Turkcan S, Gambhir SS, Pratx G. Multiscale framework for imaging radiolabeled therapeutics. Mol Pharm. 2015;12:4554-4560. 